\section{Use of a slit-lamp microscope for treating impacted facial foreign bodies in the emergency department}

\author{
Seung-Hwan Seol' ${ }^{1}$ Joonpil Cho ${ }^{2}$, Woon-Jeong Lee', Sang-Cheon $\mathrm{Choi}^{2}$ \\ 'Department of Emergency Medicine, Incheon St. Mary's Hospital, Incheon, Korea \\ ${ }^{2}$ Department of Emergency Medicine, Ajou University School of Medicine, Suwon, Korea
}

Identifying, locating, diagnosing, and treating small foreign bodies (FBs) in soft tissues is a challenge for emergency physicians in the emergency department. Additionally, potential complications owing to the remnant FBs are medico-legally significant. The efficacy of conventional imaging methods such as radiography, computed tomography, and ultrasonography are largely limited in visualizing $\mathrm{FBs}<2-\mathrm{mm}$. The slit-lamp microscope, still unfamiliar to some emergency physicians, could be used to facilitate the treatment of FBs impacted in soft tissues. In this paper, we present a case that would have been difficult to treat without the help of the slit-lamp microscope; the patient presented with numerous particulate facially impacted FBs that were too small to be observed under plain sight or with radiography. Based on our experience, the slitlamp microscope could be a useful tool for treating patients with miniscule and stubborn impacted FBs in the emergency department.

Keywords Foreign bodies; Soft tissue injuries; Therapeutics

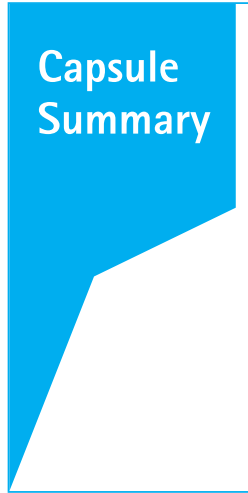

What is already known

Conventional imaging methods such as radiography, ultrasonography and computed tomography frequently fail in visualizing miniscule foreign bodies owing to the limitations of image resolution.

\section{What is new in the current study}

Using a slit lamp microscope, emergency physicians can treat the patient with miniscule and stubborn impacted foreign bodies that are in the face.
elSSN: 2383-4625

Received: 11 November 2014 Revised: 12 December 2014 Accepted: 29 December 2014

Correspondence to: Sang-Cheon Choi Department of Emergency Medicine, Ajou University School of Medicine, 206 World cup-ro, Yeongtong-gu, Suwon 443-721, Korea

E-mail:avenue59@ajou.ac.kr

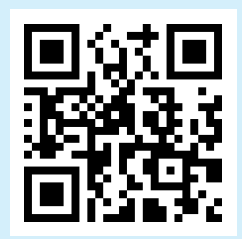

How to cite this article:

Seol SH, Cho J, Lee WJ, Choi SC. Use of a slit-lamp microscope for treating impacted facial foreign bodies in the emergency department. Clin Exp Emerg Med 2015;2(3):188-192.

This is an Open Access article distributed under the terms of the Creative Commons Attribution Non-Commercial License (http:// creativecommons.org/licenses/by-nc/3.0/). 


\section{INTRODUCTION}

Patients with stubborn or miniscule impacted foreign bodies (FBs) are often admitted to the emergency department (ED). Diagnosis and treatment of FBs could be an actual clinical challenge for emergency physicians, especially if they are invisible. ${ }^{1}$ In such cases, removing FBs is never a minor procedure, as impacted FBs may lead to many problems. ${ }^{1-5}$ They can also be very painful and irritating, with secondary infection often expected, even with tiny FBs. Additionally, inadequate treatment such as incomplete removal might lead to future cosmetic problems. ${ }^{6}$ Remnant FBs gradually decompose to attach themselves permanently to the inner dermis, creating irregular black or blue discoloration resembling a tattoo. ${ }^{6}$ The best strategy is to prevent traumatic tattooing by immediately removing the FBs before the healing process starts. ${ }^{6}$ Thus, clinicians should pay close attention to the treatment of remnant $\mathrm{FBs}$.

FB removal is better facilitated after correct identification. Impacted under the skin, FBs can be invisible; therefore, conventional imaging methods such as radiography, ultrasonography, and computed tomography (CT) are considered for improved identification and safe removal. ${ }^{1,2}$ However, conventional imaging methods frequently fail in visualizing miniscule FBs owing to the limitations of image resolution. According to Anderson et al., ${ }^{3}$ complete removal was impossible for FBs in the hands. In such cases, the slit-lamp microscope could have been helpful for clinicians identifying the FBs. In soft tissues, the focused light from a slitlamp shines on the skin, creating shadows of the impacted FBs, which could greatly improve microscopic distinguishability between the impacted FBs and the skin. The device is easily applicable to body parts including the face, hands, and fingers.

In this paper, we report a challenging case of facial FB impaction in a patient, whereby the FBs were successfully identified and removed by visualization using a slit-lamp microscope.

\section{CASE REPORT}

A 38-year-old man was admitted to the ED with stable vital signs and normal level of consciousness after he had been near the site of a high-pressure tank explosion at work. The patient was thought to have miniscule particles of cement and other materials impacted in his skin, especially in the face. Skin damage was observed on the right side of the face and neck, and the center of the chest, where numerous cement particles were presumably impacted. Additionally, the patient presented with a foot fracture, resulting from tripping while stepping backwards at the time of explosion. Facial CT scans revealed the presence of minute FBs in the dermal layers. The ophthalmology department was consulted to eliminate the possibility of ocular FBs. A corneal abrasion was observed, but no FB. Local anesthesia was administered to reduce pain. Immediate treatment consisted of thoroughly brushing out and rinsing the wounds with $2 \mathrm{~L}$ of normal saline. Visible FBs in the dermis were also removed using a no. 11 surgical blade. After removal, bleeding persisted with considerable black colored pigmentation in the affected sites, suggesting possible persistent FBs, too small to be visible to the naked eye. For further removal, a slit-lamp microscope (Topcon Slit Lamp SL-3F; Topcon Medical Systems, Oakland, NJ, USA) and a 26-gauge needle were used in order to prevent traumatic tattoos. The patient sat in front of the slit-lamp microscope as we adjusted the focus setting to $\times 16$ magnification. We increased the light intensity, and decreased the slit width. Next, we adjusted the screw of the chin rest bar for proper vertical alignment. For horizontal alignment, the face angle was adjusted to the right or left. We shone the light next to (but not directly on) the impacted area. With direct, localized light, it was too bright to see the FBs, which could be visualized as solid 3-demensional shapes defined by their shadow lines (Fig. 1A, B). We used a 26-gauge needle to access the identified areas through a wound in the skin created by the initial penetration (Fig. 1C). We confirmed the FBs by feeling them with the needle tip; larger FBs were removed through the formed passage of the wound, while the miniscule powder ones were carefully scratched off the skin surface. Approximately $50 \mathrm{FBs}$ were removed each time, with 150 removed for the three procedures in total (Fig. 1D). The removed particles were between 0.5 and $2.0 \mathrm{~mm}$. The total procedure duration was approximately 15 minutes in total. After removal, an antibiotic ointment was applied, and a moistened dressing was given; the wound recovered with no sign of traumatic tattoo or secondary infection at the time of follow-up (Fig. 2).

\section{DISCUSSION}

Misdiagnosis of FBs is a common reason for potential medico-legal issues. ${ }^{4,5}$ In soft tissues, FBs may not be found easily on routine examinations. However, complications caused by FBs in soft tissues include infection, inflammatory irritation, allergic reaction, chronic pain, and potential cosmetic issues. ${ }^{7}$ For preventing associated inflammatory irritations and infections, FBs should be removed whenever possible. This is especially true for pigmented facial FBs, which need to be immediately removed, as these embedded particles can become permanently lodged in the epidermis, leading to traumatic tattoos. ${ }^{6}$ Although some FBs may be too small to cause any inflammatory irritations, they tend to be equally hazardous. Inflammatory irritations can be triggered re- 

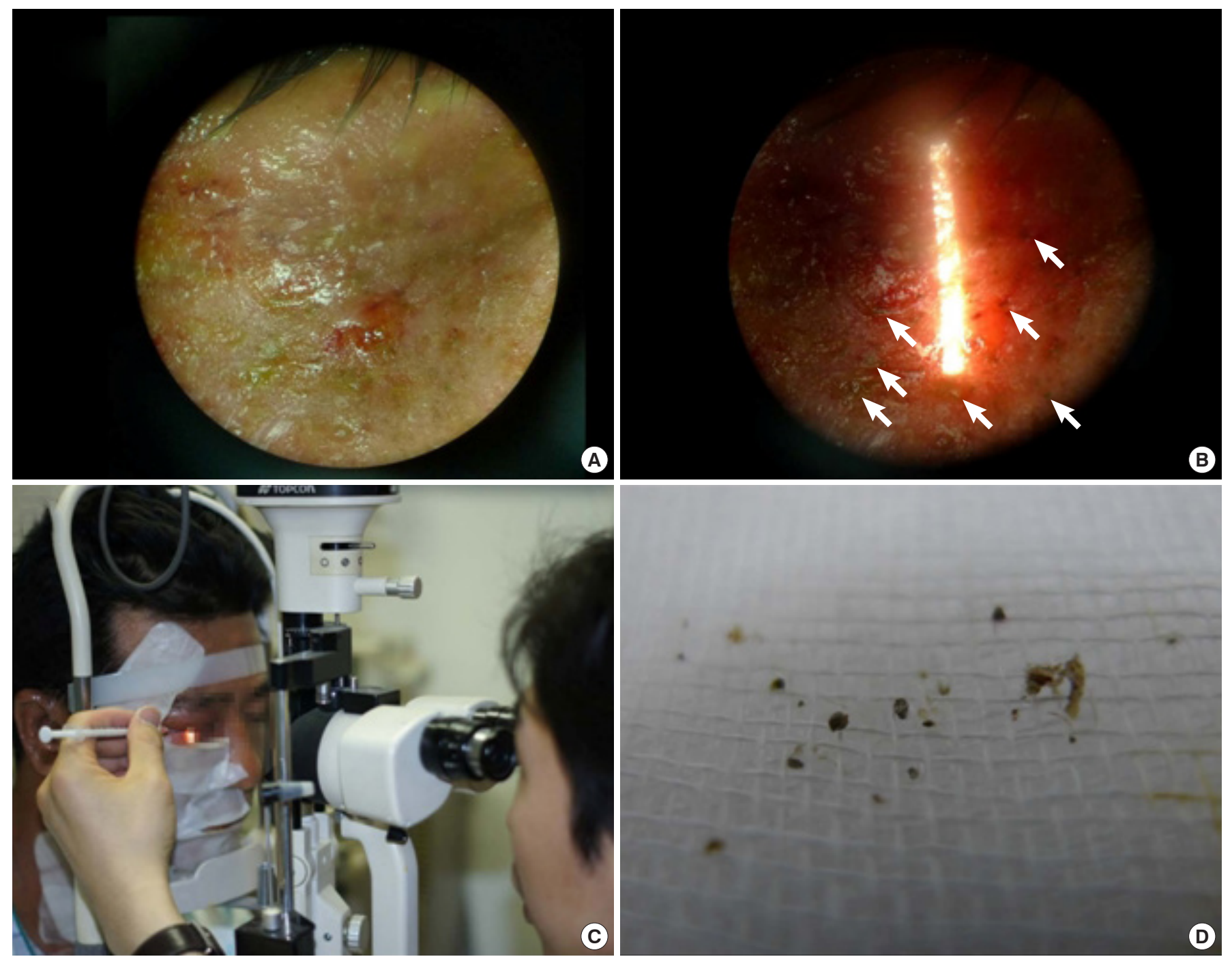

Fig. 1. Removal of foreign bodies from the face using a slit-lamp microscope. (A) Microscopic view of the impacted foreign body site (face) before the slit-lamp light beaming. (B) Microscopic view of the impacted foreign body site (face) after the slit-lamp light beaming. The shapes of embedded cement particles are shown by the slit light (white color are indicated by arrow). (C) Removal of the foreign bodies using the slit-lamp microscope. (D) The cement particles after removal (average size, 0.5 to $2.0 \mathrm{~mm}$ ).

gardless of the possibility of contamination and inflammation in the body. Remnant FBs potentially cause symptoms like infection, chronic pain, inflammation, and allergic reaction. Traumatic tattooing results from the accidental impregnation of dirt, asphalt, and gravel particles, and can occur for penetrating FBs such as pencil lead. When organ damage due to removal is an actual concern, one may consider leaving the FBs embedded. Still, where possible, complete removal is always recommended, irrespective of the FB size.

In the $E D$, when FBs are suspected clinically, plain radiography is performed. Radiopaque objects are potentially visible on radiographs. For example, for a piece of glass $>2 \mathrm{~mm}$ in size, the detection rate is approximately 50\% to $60 \%$. CT is 100 times more sensitive than plain radiography for distinguishing low density $\mathrm{FBs}^{2,8}$ The main drawbacks of CT are cost and increased level of radiation exposure. ${ }^{2,8}$ Magnetic resonance imaging can also differentiate radiolucent objects, but it is costly and unavailable in most EDs. ${ }^{9}$ Ultrasonography is a bedside device allowing onsite confirmation with no radiation, and successful FB removal using this method has been reported. ${ }^{10-12}$ In addition, real-time video monitoring in ultrasonography could be advantageous. ${ }^{12,13}$ However, successful FB removal will depend on the operator's skills, and removals of FBs $<2 \mathrm{~mm}$ in size are not assisted using ultrasonography. ${ }^{12,13}$ Objects $<2 \mathrm{~mm}$ in size are difficult to find and remove using conventional methods. ${ }^{13}$ Moreover, the challenge is greater if the site is covered with scars, or clotted with blood or 


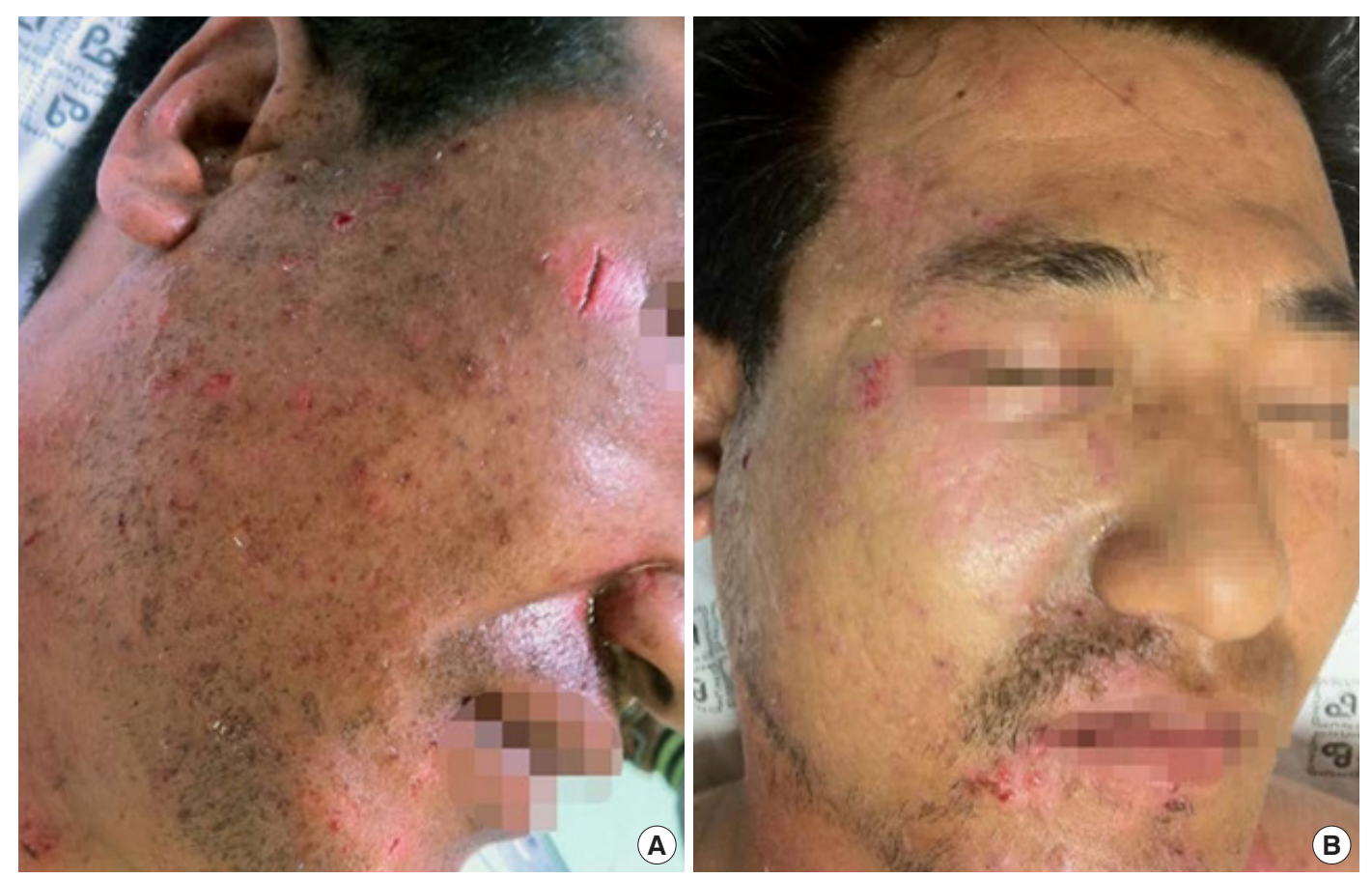

Fig. 2. Before and after foreign body removal from the face. (A) Before the foreign body removal, when hundreds of small cement particles were embedded in the face. (B) After foreign body removal from the patient's forehead, eye sockets, and ala of the nose. The impacted site recovered with no traumatic tattoo or secondary infection.

body fluid. There is an urgent need for improvement in identifying the accurate location of the FBs impacted in soft tissues in order to assist in effective removal.

The slit-lamp microscope is for ophthalmology examinations and procedures. ${ }^{14}$ The slit-lamp shines a thin, long light on the human eye so as to reveal the cornea and crystal line. Optical sectioning is performed by the slit-lamp, and the section is observed under the microscope unit. By adjusting the light focus and incidence angle, one can observe the eyeball from the conjunctiva to the front of the vitreous. We employed this method for examining impacted FB to expand its applicability to cover the face. In our case, FBs were best observed under $\times 10$ or $\times 16$ magnification. The device currently allows for the adjustment of light intensity, direction, size, and width from a slit-lamp. After successful visualization in the microscope, we removed the FBs using a sharp sterilized tool, and avoided unnecessary damages or bleeding.

The present report has several limitations. First, the efficiency of the removal method is yet unknown. Removal of FBs may not result in better cosmetic results than those by other treatments. However, considering the issues of potential medico-legal problems and traumatic tattoos, we believe that this method is worth using in patients in the ED. Second, the slit-lamp microscope may not be available in some EDs. Therefore, one may not apply the method for patients with impacted FBs in all emergency depart- ment settings. Furthermore, considerable time will be needed for the procedure if several FBs are impacted. The ED is usually crowded with patients needing emergency treatment. Therefore, conducting the procedure in the ED should take into consideration the human resources available at that time.

To our knowledge, there is only one report regarding the application of the slit-lamp microscope for impacted soft tissue FBs. ${ }^{15}$ For some emergency physicians, the slit-lamp microscope could be an unfamiliar examination tool, but it will be important for helping remove stubborn FBs. We suggest that emergency physicians expand their knowledge of the slit-lamp microscope and consider applying it for removing difficult FBs such as those that are miniscule, stubborn, or invisible. Proper and immediate removal of FBs is undoubtedly associated with preventing complications such as infection and permanent tattoos by embedded FBs requiring secondary surgical operation or laser removal. Removing miniscule and stubborn FBs impacted in soft tissues may be a minor procedure, but it can be a great challenge demanding an immediate response from emergency physicians.

\section{CONFLICT OF INTEREST}

No potential conflict of interest relevant to this article was reported. 


\section{ACKNOWLEDGMENTS}

The authors thank all the members of the Society for Clinical Procedure and Education-Eye Emergency Procedures Division, whose support made this report possible.

\section{REFERENCES}

1. Flom $L L$, Ellis GL. Radiologic evaluation of foreign bodies. Emerg Med Clin North Am 1992;10:163-77.

2. Ginsburg MJ, Ellis GL, Flom LL. Detection of soft-tissue foreign bodies by plain radiography, xerography, computed tomography, and ultrasonography. Ann Emerg Med 1990;19: 701-3.

3. Anderson MA, Newmeyer WL 3rd, Kilgore ES Jr. Diagnosis and treatment of retained foreign bodies in the hand. Am J Surg 1982;144:63-7.

4. Karcz A, Holbrook J, Auerbach BS, et al. Preventability of malpractice claims in emergency medicine: a closed claims study. Ann Emerg Med 1990;19:865-73.

5. Karcz A, Korn R, Burke MC, et al. Malpractice claims against emergency physicians in Massachusetts: 1975-1993. Am J Emerg Med 1996;14:341-5.

6. Kent KM, Graber EM. Laser tattoo removal: a review. Dermatol Surg 2012;38:1-13.

7. Lammers RL. Soft tissue foreign bodies. Ann Emerg Med 1988;
17:1336-47.

8. Mizel MS, Steinmetz ND, Trepman E. Detection of wooden foreign bodies in muscle tissue: experimental comparison of computed tomography, magnetic resonance imaging, and ultrasonography. Foot Ankle Int 1994;15:437-43.

9. Russell RC, Williamson DA, Sullivan JW, Suchy H, Suliman 0. Detection of foreign bodies in the hand. J Hand Surg Am 1991; 16:2-11.

10. Shiels WE 2nd, Babcock DS, Wilson JL, Burch RA. Localization and guided removal of soft-tissue foreign bodies with sonography. AJR Am J Roentgenol 1990;155:1277-81.

11. Bray PW, Mahoney JL, Campbell JP. Sensitivity and specificity of ultrasound in the diagnosis of foreign bodies in the hand. J Hand Surg Am 1995;20:661-6.

12. Manthey DE, Storrow AB, Milbourn JM, Wagner BJ. Ultrasound versus radiography in the detection of soft-tissue foreign bodies. Ann Emerg Med 1996;28:7-9.

13. Levine MR, Gorman SM, Young CF, Courtney DM. Clinical characteristics and management of wound foreign bodies in the ED. Am J Emerg Med 2008;26:918-22.

14. Yoon SK, Choi SC, Kim GW, Min YG, Cho JP. Corneal foreign body removal by emergency physicians. J Korean Soc Emerg Med 2003;14:1-4.

15. Seol SH, Oh S, Choi SC. Young man with pain in finger. Ann Emerg Med 2015;65;e3-4. 